\title{
Bullous cellulitis with Cryptococcus neoformans
}

\author{
D. Vuichard $\cdot$ A. Conen $\cdot$ M. Brenner • \\ P. Itin · U. Flückiger
}

Received: 14 December 2010/Accepted: 12 January 2011/Published online: 8 February 2011

(C) Urban \& Vogel 2011

\begin{abstract}
A 79-year-old immunocompromised pigeon farmer presented with a 2-week history of a well-demarcated redness and swelling of his left hand spreading to the upper arm. His medical history was remarkable for untreated myelodysplastic syndrome. Despite antibiotic treatment for assumed bacterial cellulitis, he developed progressive erythema, partial skin necrosis, and hemorrhagic epidermal bullae (Fig. 1a). Examination of deep skin biopsy specimens revealed granulomatous inflammation and encapsulated yeasts compatible with Cryptococcus neoformans (Fig. 1b, c), which was subsequently confirmed in microbiological culture. Fluconazole therapy was initiated, and several surgical debridements were performed. The patient is currently receiving antifungal treatment on an outpatient basis and is in good health.
\end{abstract}

D. Vuichard $(\square)$

Division of Infectious Diseases and Hospital Epidemiology,

University Hospital Basel, Petersgraben 4, 4031 Basel,

Switzerland

e-mail: vuichardd@uhbs.ch

\section{A. Conen}

Division of Infectious Diseases and Hospital Epidemiology, Department of Internal Medicine, Cantonal Hospital Aarau, Aarau, Switzerland

\section{Brenner · P. Itin}

Department of Dermatology, University Hospital Basel,

Basel, Switzerland

\section{U. Flückiger}

Infectious Diseases, Hirslanden Clinic Aarau,

Aarau, Switzerland

\section{U. Flückiger}

Faculty of Medicine, University Basel, Basel, Switzerland
The skin is the third most common organ affected in C. neoformans infections, after the lung and central nervous system. Although direct inoculation has been described, cutaneous cryptococcal infection most commonly results from the hematogenous spread of inhaled organisms. Cryptococcal cellulitis may mimic bacterial infection in both appearance and rapidity of onset. Thus, in cases of soft tissue infections not responding to broad-spectrum antibiotics, it is of utmost importance that the clinician consider the possibility of this yeast as causal agent, especially in immunocompromised patients. Obtaining skin biopsies for culture and histopathologic examination and the prompt administration of an antifungal agent is crucial to successful treatment. In a case involving immunosuppression, a prolonged treatment course of 6-12 months has to be considered, even in the absence of clinical or microbiological signs of dissemination.

Conflict of interest None. 
Fig. 1 a Left arm with

erythematous, partially necrotic skin and hemorrhagic bullae, b periodic acid-Schiff stain $(\times 40)$ of skin biopsy showing an encapsulated yeast (arrow), c multiple encapsulated yeasts on Grocott stain $(\times 40)$ of a tissue specimen
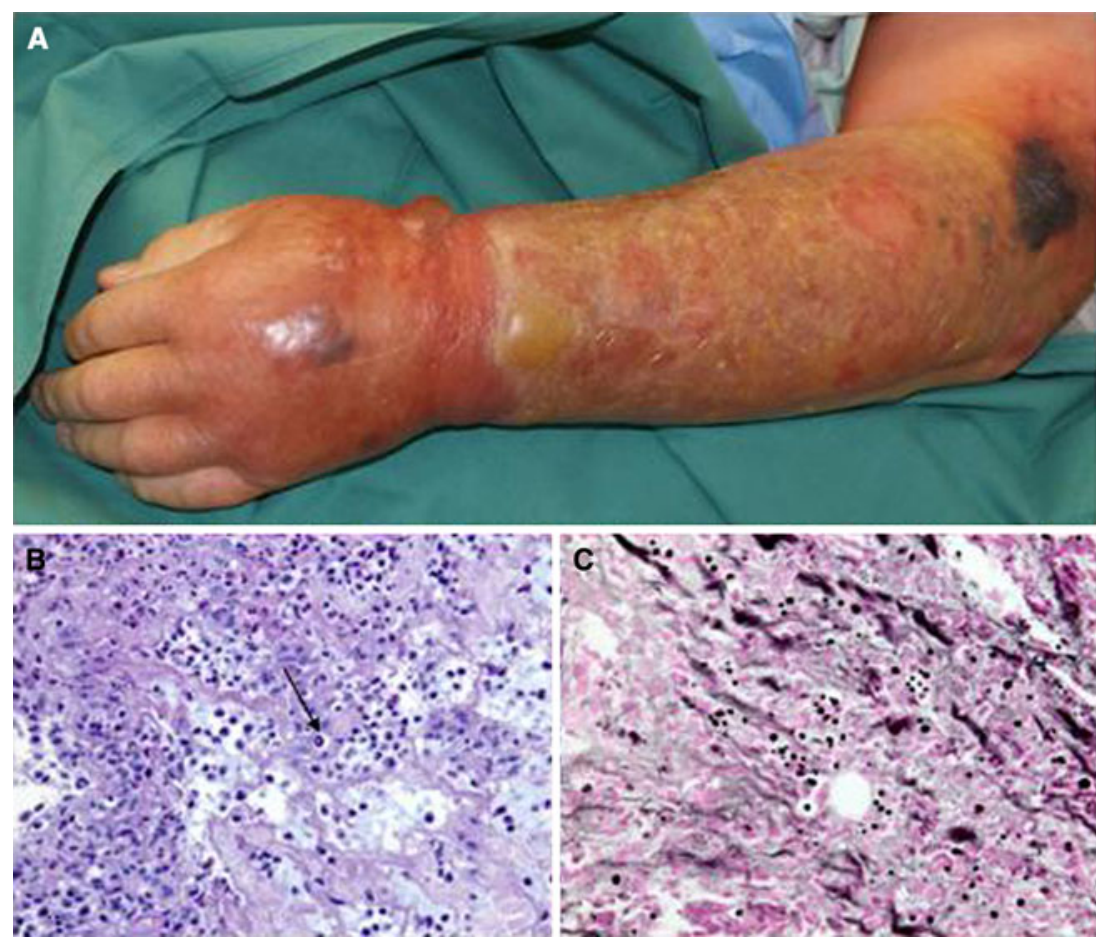